\title{
Follow-up on pediatric patients with bronchiolitis obliterans treated with corticosteroid pulse therapy
}

\author{
Silvia Onoda Tomikawa ${ }^{1,3^{*}}$, Fabíola Villac Adde ${ }^{1}$, Luiz Vicente Ribeiro Ferreira da Silva Filho ${ }^{1}$, Claudio Leone ${ }^{2}$ \\ and Joaquim Carlos Rodrigues ${ }^{1}$
}

\begin{abstract}
Background: Bronchiolitis obliterans $(\mathrm{BO})$ is a rare but severe disease in children. Currently, there is no consensus on the treatment for $\mathrm{BO}$ with respect to the systemic use of corticosteroids. Here we report on the follow-up of children with a diagnosis of $\mathrm{BO}$ who were treated with corticosteroid pulse therapy.

Methods: Forty patients fulfilling the BO diagnosis criteria were treated with methylprednisolone pulse therapy in monthly cycles until clinical improvement. After the pulse therapy began, we analyzed the clinical and laboratory data at intervals. Statistical analyses were performed using non-parametric tests to compare repeated measures (Friedman, Wilcoxon) or paired nominal data (McNemar) $(a=5 \%)$.

Results: The frequency of wheezing exacerbations and hospitalizations was reduced ( $p=0.0042$ and $p<0.0001$, respectively) and oxygen saturation improved $(p=0.0002)$ in the pulse therapy-treated patients. Prolonged oral corticosteroid therapy was discontinued in $83 \%$ of these patients. The mean Z-score length for age improved from -1.08 to -0.63 , and the mean Z-score weight for age improved from -0.91 to -0.59 . The adverse effects during the infusion were temporary and none were serious.

Conclusions: Our data suggest that pulse corticotherapy could be a safe alternative to prolonged systemic oral corticotherapy in children with $\mathrm{BO}$, thus minimizing the adverse effects of the oral therapy. New prospective controlled studies are required to confirm this proposition.
\end{abstract}

Keywords: Bronchiolitis obliterans, Pulse therapy, Methylprednisolone

\section{Background}

Bronchiolitis obliterans (BO) is a rare form of chronic obstructive lung disease that follows an infection-induced injury to the lower respiratory tract. This condition results in partial or complete obliteration of the small airways [1-3]. Although the first description of clinical cases of bronchiolitis obliterans is credited to Lange in 1901, many aspects of this disease remain unknown [4-6].

Bronchiolitis obliterans is an uncommon disease, but a growing number of reports in recent decades have resulted in a greater awareness of the condition [2,3]. Although

\footnotetext{
*Correspondence: silviatomi@yahoo.com.br

${ }^{1}$ Pediatric Pulmonology Division, Instituto da Criança, Hospital das Clínicas,

University of São Paulo, Avenida Dr Enéas de Carvalho Aguiar, 647, CEP 05403-000 São Paulo, SP, Brazil

${ }^{3}$ Rua Bianchi Bertoldi, 166 apt 101, CEP: 05422-070 São Paulo, SP, Brazil Full list of author information is available at the end of the article
}

there are no worldwide studies of the prevalence of postinfectious $\mathrm{BO}$, it has mainly been reported in the southern hemisphere (Argentina, Chile, southern Brazil, Australia and New Zealand) [1,2,7].

No universally accepted protocol has been established for the treatment of $\mathrm{BO}[6,8]$. Corticosteroid therapy seeks to modify the fibroblastic response in the early phase of illness. This approach is based on a study conducted in 1958 in which steroids were administered to rabbits to prevent development of $\mathrm{BO}$ [9]. Others studies have reported variable efficacy of corticosteroid therapy in patients with $\mathrm{BO}[1,5,10]$.

Methylprednisolone intravenous pulse therapy has been proposed to enhance the therapeutic effects and reduce the side effects of corticotherapy, and it is an alternative treatment for patients with more severe disease 
$[1,7,10]$. The aim of the present study was to review the clinical, laboratory and radiological data collected before and after treatment from patients with bronchiolitis obliterans who were treated with methylprednisolone pulse therapy.

\section{Methods}

Forty children of both genders aged 5 months to 13 years and diagnosed with $\mathrm{BO}$ were followed up retrospectively at the Pediatric Pulmonology Unit of the Instituto da Criança, Hospital das Clínicas, University of São Paulo. The diagnosis of BO was made according to clinical criteria (acute lower respiratory tract infection associated with persistent obstructive respiratory disease after the initial event, which was not responsive to treatment for a period longer than 6 weeks), the results of high resolution computerized tomography of the thorax (mosaic pattern and/or bronchiectasis) and in some patients, with lung biopsies (obliterative bronchiolitis). Other causes of chronic obstructive pulmonary disease, including cystic fibrosis, bronchopulmonary dysplasia, pulmonary tuberculosis, $\alpha 1$-antitrypsin deficiency, immunodeficiencies, aspiration of foreign bodies and cardiac diseases, were excluded.

The cases included all patients who received at least six cycles of treatment with intravenous corticosteroid pulse therapy (methylprednisolone $30 \mathrm{mg} / \mathrm{kg} / \mathrm{BW}$ per day for three days) from 1996 to 2007. The criteria for beginning corticosteroid pulse therapy in the patients with $\mathrm{BO}$ was the severity of the condition as indicated by difficulty in the removal of the prolonged oral corticosteroids and/or hypoxemia requiring home oxygen therapy.

This pulse regimen (30 $\mathrm{mg} / \mathrm{kg} /$ day for 3 days) is already used in other childhood diseases such as renal and rheumatologic diseases [11-15]. The corticosteroid dose of $10 \mathrm{mg} / \mathrm{kg} /$ day is classically used in restrictive interstitial lung diseases, but we chose the $30 \mathrm{mg} / \mathrm{kg} /$ day dose due to the clinical severity of the disease in our patients. Recently, the $30 \mathrm{mg} / \mathrm{kg} /$ day pulse regimen has also been used in children with pneumonia caused by Mycoplasma pneumoniae [16].

The initial cycles of pulse therapy were repeated on a monthly basis. As clinical improvement was demonstrated, the cycle interval was increased to two months, and pulse therapy was later discontinued. All of the clinical and radiological data regarding the patients' medical histories, such as duration of gestation, breast feeding, parental smoking and family history of asthma, were obtained from previous medical records.

\section{Statistical analysis}

Clinical and laboratory data were analyzed at intervals after the pulse therapy began, and non-parametric statistical tests were used to compare repeated measures
(Friedman, Wilcoxon) or paired nominal data (McNemar). A Dunn post-test with statistical significance defined as $\alpha=5 \%$ was performed for multiple comparisons.

\section{Ethical approval}

The ethics committee for analysis of clinical research projects of Hospital das Clinicas - University of São Paulo (CAPpesq) approved this research, protocol number 0695/07.

\section{Results}

\section{Characteristics of the patients}

Table 1 shows the characteristics and history of the 40 patients. Our study sample consisted of 30 (75\%) boys and 10 (25\%) girls, ranging in age (at diagnosis) from 5 months to 13 years. At diagnosis, the time elapsed since the episode of acute illness was between 1 and 134 months (mean 24.6 months, median 12.5 months). The average number of previous hospitalizations was 4 per year. Twenty-one patients (52.5\%) required mechanical ventilation during a previous hospitalization, and 8 (20\%) needed domiciliary oxygen therapy.

Table 2 shows that the most common symptoms and signs of the 40 patients diagnosed with $\mathrm{BO}$ were wheezing, dyspnea and cough.

Table 3 shows that the most frequent findings of the chest HRCT were a mosaic perfusion pattern and bronchial wall thickening.

\section{Etiology}

The presumed etiology was post-infectious in 37 patients. Because viral identification for the acute bronchiolitis was not performed in all patients referred to the hospital, viral identification by paired serology was only available for 5 patients: 2 with adenovirus, 1 with respiratory syncytial virus (RSV), and 2 with adenovirus plus RSV. The other 32 patients had typical histories of previous severe acute bronchiolitis (most with hospitalization and even requiring mechanical ventilation) and after this event, the airway obstruction persisted for a period longer than 6 weeks. On this basis, these patients were considered to have post-infectious $\mathrm{BO}$.

Two patients had histopathological findings consistent with obliterative bronchiolitis and indicative of aspiration syndrome. One patient had a history of acute viral bronchiolitis with persistence of airway obstruction and severe gastro-esophageal reflux. For this patient, we considered that viral infection and aspiration could be both associated with the etiology.

\section{Pathologic findings}

Open lung biopsies were performed in 18 patients. In 14 of these, the pathologic findings were consistent with obliterative bronchiolitis. The histological patterns were: 
Table 1 Characteristics and histories of $\mathbf{4 0}$ patients with bronchiolitis obliterans

\begin{tabular}{lll}
\hline Characteristics $(\mathbf{n}=\mathbf{4 0})$ & & \\
\hline Gender & Male $=30(75 \%)$ & Female $=10(25 \%)$ \\
Gestational age & Full term $=35(87.5 \%)$ & Premature $=5(12.5 \%)$ \\
Breast-feeding & $<3$ months $=21(52.5 \%)$ & $3-6 \mathrm{~m}=17(42.5 \%)$ \\
Family history of asthma & $28(70 \%)$ & \\
Parental smoking & $22(55 \%)$ & \\
\hline History ( $\mathbf{n}=\mathbf{4 0})$ & mean 7.4, median $4.5(0-48$ months) \\
\hline Age at first wheezing & mean 18.3, median 9.5 (0-150 months) \\
Age at onset of persistent wheezing & mean 42.9, median 25 (5-156 months) \\
Age at diagnosis & mean 24.6, median 12.5 (1-134 months) \\
Onset of disease/diagnosis interval & $24(60 \%)$ \\
Recurrent pneumonia episodes & mean 4 times per year, median 2.8 times per year (0-10 times per year) \\
Hospitalization & $21(52.5 \%)$ \\
Mechanical ventilation assistance & $8(20 \%)$ \\
Domiciliary oxygen therapy & $23(57.5 \%)$ \\
Prolonged oral corticosteroid therapy & $19(47.5 \%)$ \\
Inhaled corticosteroid therapy &
\end{tabular}

constrictive in 13 patients; proliferative in 1 patient; and inconclusive in 4 patients.

\section{Lung function measurements}

Most patients did not perform a lung function test because they were under 6 years of age. Spirometry, including a bronchodilator response test, was performed in only 8 patients (ages 6-14, mean age 9.6 years). The means of the initial spirometry parameters, expressed as percentages of the predicted values, were: FVC: $77.2 \%$; $\mathrm{FEV}_{1}$ : 55.3\%; $\mathrm{FEV}_{1} / \mathrm{FVC}: 67.4 \%$ and $\mathrm{FEF}_{25-75 \%}$ : $35.7 \%$. Three patients responded significantly to bronchodilation

Table 2 Symptoms and signs at time of diagnosis

\begin{tabular}{ll}
\hline Characteristics $(\mathbf{n}=\mathbf{4 0})$ & \\
\hline Symptoms & $23(57.5 \%)$ \\
Persistent cough & $26(65 \%)$ \\
Dyspnea & $40(100 \%)$ \\
Persistent wheezing & $9(22.5 \%)$ \\
Cyanosis (reported episodes) & \\
Physical examination & $23(57.5 \%)$ \\
Increased anterior-posterior & \\
chest diameter & $8(20 \%)$ \\
Cushing syndrome-like aspect & $7(17.5 \%)$ \\
Clubbing fingers & $2(5 \%)$ \\
Watch glass nails & Diffuse crackles = 24 (60\%) \\
Pulmonary auscultation & Localized crackles = $7(17.5 \%)$ \\
& Wheezing = 33 (82.5\%) \\
\hline
\end{tabular}

according to criteria from the American Thoracic Society and the European Respiratory Society [17].

Because patient follow-up was performed for many years, the first spirometry results were available for 24 patients (age 6-8 years, mean 7.2 years) after pulse therapy (mean time 27 months), with parameter means as follows: FVC: $66.8 \% ; \mathrm{FEV}_{1}$ : 47.7\%; $\mathrm{FEV}_{1} / \mathrm{FVC}$ : $65.6 \%$ and $\mathrm{FEF}_{25-75 \%}: 26.6 \%$. Five of these patients had a response to bronchodilation according the American Thoracic Society and the European Respiratory Society criteria [17].

\section{Follow-up}

\section{Clinical follow-up}

The patients were followed for an average of 51.6 months (ranging from 23 to 93 months), and 24 patients are still being observed (Table 4 ). The total number of treatment cycles with pulse therapy ranged from 6 to 40 (mean 20.2, median 18.5).

\section{Table 3 HRCT* findings in children with bronchiolitis} obliterans

\begin{tabular}{ll}
\hline HRCT findings $(\mathbf{n}=\mathbf{4 0})$ & \\
\hline Mosaic perfusion pattern & $29(72.5 \%)$ \\
Bronchial wall thickening & $18(45 \%)$ \\
Atelectasis & $16(40 \%)$ \\
Alveolar filling & $12(30 \%)$ \\
Bronchiectasis & $11(27.5 \%)$ \\
Hyperinflation & $5(12.5 \%)$ \\
Air trapping & $5(12.5 \%)$ \\
Swyer-James-MacLeod syndrome & $1(2.5 \%)$ \\
\hline
\end{tabular}

*HRTC- High resolution computed tomography. 
Table 4 Characteristics of the clinical follow-up of patients with BO

\begin{tabular}{ll}
\hline Characteristics $(\mathbf{n}=\mathbf{4 0})$ & \\
\hline $\begin{array}{l}\text { Age at beginning of follow-up } \\
\text { Follow-up period }\end{array}$ & mean 40.9, median 25 (6-186 months) \\
$\begin{array}{l}\text { Age at beginning of } \\
\text { pulse therapy }\end{array}$ & mean 50.5, median 31.5 (6-180 months) \\
$\begin{array}{l}\text { Onset of disease/pulse } \\
\text { therapy interval }\end{array}$ & mean 32.1, median 18.5 (2-142 months) \\
$\begin{array}{l}\text { Pulse therapy cycles } \\
\text { Pulse therapy period }\end{array}$ & mean 20.2, median 18.5 (6-40 cycles) \\
\hline
\end{tabular}

In addition to the systemic corticosteroid therapy, all 40 patients received inhaled corticosteroids to reduce airway hyperreactivity for the entire follow-up period. Other medications used by the patients during the pulse therapy period included: long-term beta-2-agonist bronchodilators in 23 patients (57.5\%); anti-gastroesophageal reflux drugs in 23 (57.5\%); antibiotic prophylaxis in 19 (47.5\%); anti-hypertensive drugs in 21 (52.5\%); digoxin in $2(5 \%)$; and diuretics in $13(32.5 \%)$.

\section{Development}

The clinical data were analyzed at intervals before and after pulse therapy began (every 6 months, before and after therapy). The frequency of the wheezing exacerbations (Figure 1a) was significantly reduced after 24 months of pulse therapy relative to the baseline ( 6 months before therapy) $(\mathrm{p}=0.0042)$. The frequency of hospitalization after 18 months of pulse therapy (Figure $1 \mathrm{~b}$ ) was significantly reduced compared to the baseline ( 6 months before therapy) $(\mathrm{p}<0.0001)$.

The initial transcutaneous oxygen saturation measurements (Figure 1c and d) showed hypoxemia (<95\%) in 29 patients $(n=35)$. The level of oxygen saturation improved compared to the baseline in the first $(\mathrm{p}=0.0002)$ and second years $(\mathrm{p}=0.0005)$ following pulse therapy.

The mean length-for-age Z-score (Figure 2a) improved compared to the baseline and after the end of pulse therapy, from -1.08 to $-0.63(\mathrm{p}=0.015)$. The mean weight-forage Z-score (Figure 2b) was also improved at the end of pulse therapy compared to the baseline: from -0.91 to -0.59 $(\mathrm{p}=0.039)$.

Spirometry was performed in 8 patients before pulse therapy. Only 5 patients repeated the exam after 1 year of treatment. The mean of the spirometric results 1 year before and 1 year after treatment were, respectively: FVC: 72.6\%/78.9\%; $\mathrm{FEV}_{1}$ : 48.8\%/52.6\%; $\mathrm{FEV}_{1} / \mathrm{CVF}: 62.5 \% / 63 \%$ and $\mathrm{FEF}_{25-75 \%}: 24.2 \% / 22.9 \%$. Only an evaluation of the means is presented because it was not possible to perform a statistical analysis due the small number of patients.

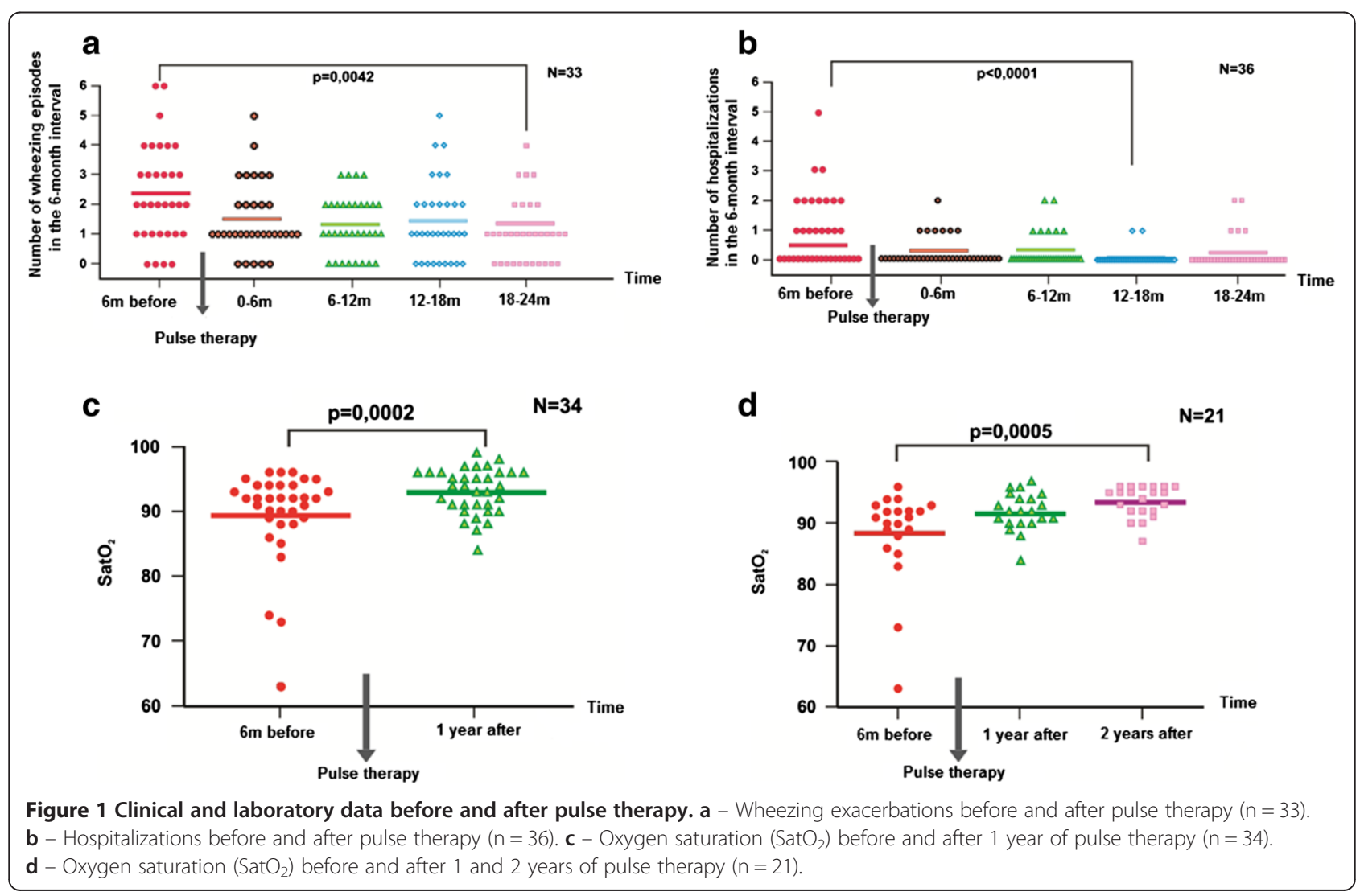



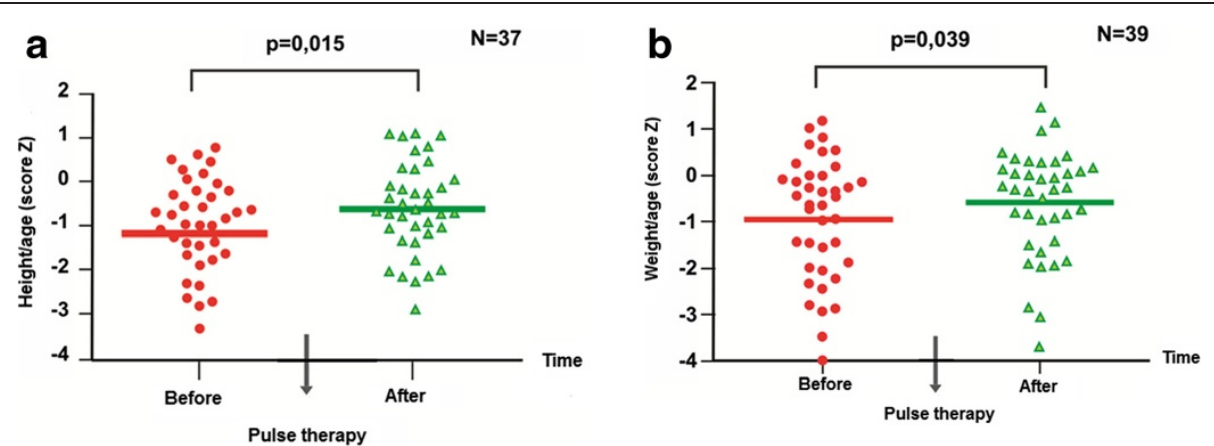

Figure 2 Height and weight before and after pulse therapy. $\mathbf{a}$ - Height-for-age (Z-score) before and after pulse therapy $(n=37)$. $\mathbf{b}-$ Weightfor-age (Z-score) before and after pulse therapy $(n=39)$.

During follow-up, chest tomography was repeated in 37 patients, and the initial abnormalities, such as bronchiectasis, bronchial wall thickening and mosaic perfusion patterns, remained in all of these patients. Normalization of tomographic alterations was not achieved during the follow-up period.

Before pulse therapy, 23 patients received continuous oral corticosteroid therapy. However, 24 months after starting pulse therapy, 19 patients (83\%) were able to discontinue the oral corticosteroids at an average of 12.4 months (Table 5). The difference between the frequency of use of oral corticosteroids before and 24 months after pulse therapy is statistically significant (McNemar test, $\mathrm{p}<0.001$ ).

Prior to pulse therapy, 20 patients required supplemental oxygen therapy due to low oxygen saturation levels on room air and/or cor pulmonale (Table 5). The hypoxemia improved during follow up, and as a result, twenty-four months after starting the pulse therapy, the administration of oxygen therapy was totally discontinued in 5 patients (25\%), while oxygen therapy was modified from full-time to overnight-only in another 5 patients $(25 \%)$. The difference between the frequency of use of oxygen before and 24 months after pulse therapy is statistically significant (McNemar, $\mathrm{p}<0.029$ ).

Table 5 Laboratory evaluation before and after pulse therapy

\begin{tabular}{lll}
\hline Characteristics $(\mathbf{n}=\mathbf{4 0})$ & Before & After $\mathbf{2 4 ~} \mathbf{~}$ \\
\hline Cor pulmonale & $27^{*}(71 \%)$ & $11^{* *}(47 \%)$ \\
Oral prolonged corticotherapy & $23(58 \%)$ & $4(10 \%)$ \\
Oxygen therapy & & \\
Full-time & $12(30 \%)$ & $4(10 \%)$ \\
Overnight & $8(20 \%)$ & $11(28 \%)$ \\
\hline
\end{tabular}

*2 patients with no echocardiogram in their medical records.

** of 27 patients with an abnormal echocardiogram, 23 repeated the exam.
Echocardiograms showed evidence of pulmonary hypertension - mean pulmonary artery pressure above 25 $\mathrm{mmHg}$ at rest or above $30 \mathrm{mmHg}$ in exercise, or systolic pulmonary artery pressure above $30 \mathrm{mmHg}$ at rest or above $35 \mathrm{mmHg}$ in exercise [18] - in 27 patients before pulse therapy. All patients with abnormal echocardiograms were referred to the Pediatric Cardiology Unit for evaluation. The cardiologist performed a physical exam with echocardiography and electrocardiography. Twentyfour months after starting pulse therapy, the cor pulmonale abnormalities disappeared in 12 patients (Table 5). The difference between the frequency of pulmonary hypertension before and 24 months after pulse therapy is statistically significant (McNemar, $\mathrm{p}<0.001$ ).

\section{Comparison of patients with and without prolonged oral corticosteroids}

Because 23 patients were already on continuous oral corticosteroid therapy at the beginning of the pulse therapy treatment and 17 were not, we compared the outcomes of the two groups.

Survival curves to compare the pulse duration of the patients who were on prolonged oral corticosteroid therapy and of the patients who were not given this therapy were produced (Figure 3a). The difference between the curves resulted not statistically significant (Log-rank test, $\mathrm{p}=0.297$.

Analysis of the frequency of wheezing exacerbations and hospitalizations showed a decrease in the number of hospitalizations in the group with prolonged oral corticosteroid therapy after pulse therapy compared to the baseline $(6$ months before therapy) $(\mathrm{p}<0.001)$ (Figure 3b). Furthermore, the median number of hospitalizations before pulse therapy differed from the medians at all other times (6, 12, 18 and 24 months). However, no statistically significant differences among the median numbers of exacerbations were found $(\mathrm{p}=0.127)$ (Figure 3c). 


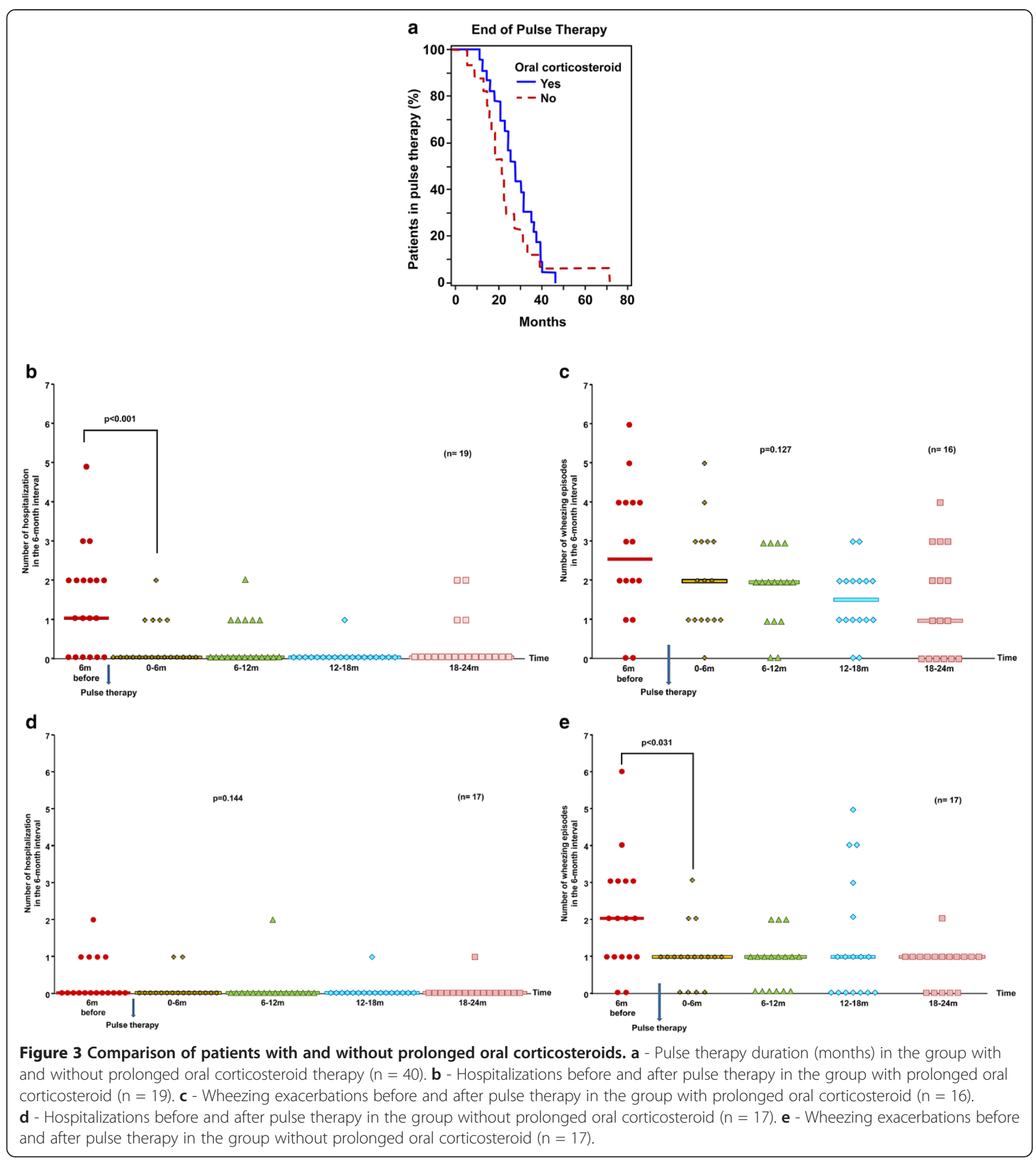

After pulse therapy, the group without prolonged oral corticosteroid therapy had a decrease in the number of exacerbations when compared to the baseline ( 6 month before therapy) $(\mathrm{p}<0.031)$ (Figure 3e). Additionally, the median number of exacerbations before pulse therapy differed from the medians at all other times (6, 12, 18 and 24 months). However, no statistically significant differences among the median numbers of hospitalizations were found $(p=0.144)$ (Figure 3d).

\section{Adverse effects}

\section{Adverse effects during the infusion}

All of the adverse effects during methylprednisolone intravenous infusion were temporary (Table 6). The most common adverse effect was hyperglycemia $(9.1 \%$ of 
Table 6 Adverse effects of pulse therapy

\begin{tabular}{ll}
\hline Adverse effects (number of cycles $=\mathbf{8 0 8}$ ) \\
\hline Hyperglycemia $>200 \mathrm{mg} / \mathrm{dl}^{*}$ & 74 episodes ( $9 \%$ of cycles) \\
Hypernatremia & 14 episodes ( $1.7 \%$ of cycles) \\
Acute hypertension & 19 episodes ( $2.3 \%$ of cycles)
\end{tabular}

*patients did not fast because the visits to the day hospital were after lunch time.

cycles), but none of these patients developed diabetes mellitus. Of the 19 episodes of acute hypertension, 10 occurred in patients with a prior diagnosis of systemic hypertension.

\section{Adverse effects of prolonged corticosteroid therapy}

Bone mineral density was measured in 25 patients. The results were normal in 13, abnormal in 11 and borderline in 1 case. The patients who were considered to be abnormal or borderline were referred to the pediatric rheumatology unit, where 8 were diagnosed with osteoporosis (1 with hypophosphataemic rickets and salt-losing tubulopathy) and 4 were treated with alendronate.

Fundoscopy examinations were performed in 28 patients, and the results were abnormal in 4 . Two presented systemic-hypertension-related lesions, 1 presented with a toxoplasmosis scar and 1 developed a cataract 1 year after the termination of pulse therapy. Urinary tract ultrasounds were performed in 37 patients and were found to be abnormal in 7 patients. Two presented with nephrolithiasis, 4 with nephrocalcinosis and 1 with a small pyelocaliceal dilatation.

None of the patients were diagnosed with diabetes mellitus. Three were referred to the pediatric endocrinology unit due to multiple hyperglycemic episodes. Each of these patients had normal levels of fasting plasma glucose and glycosylated hemoglobin.

Sixteen patients were diagnosed with systemic hypertension, but 9 of these were diagnosed before beginning pulse therapy.

\section{Discussion}

Bronchiolitis obliterans has a number of causes, including connective tissue disorders, occupational inhalation injuries, hypersensitivity pneumonitis, drugs, radiation, Stevens-Johnson syndrome and lung, heart/lung or bone marrow transplantation $[1,3,4]$.

In childhood, $\mathrm{BO}$ occurs most commonly after a severe lower respiratory tract infection and is considered a longterm sequela of viral infections $[1,5,19]$. Several agents have been associated with the development of postinfectious BO, such as adenovirus (types 3, 7 and 21), respiratory syncytial virus (RSV), influenza, parainfluenza, measles and Mycoplasma pneumoniae [5,10,20]. Adenovirus infection and the severity of acute illness (hospital days, intensive care unit admission, mechanical ventilation, oxygen use, corticosteroid treatment and $\beta 2$ agonist administration) are the most significant risk factors for developing post-infectious $\mathrm{BO}$ in children $[2,19,21]$. This is consistent with our study, in which the presumed etiology was post-infectious in 37 patients, based on histories of prior infection and/or positive viral serology findings.

The diagnosis of $\mathrm{BO}$ was based on clinical criteria, findings on HRTC scans and/or open lung biopsies [7,10,22-25]. Although lung biopsy is the gold standard for diagnosis, the sensitivity is low due to the heterogeneous distribution of airway involvement throughout the lung parenchyma $[1,3,10]$. Lung biopsies are not usually needed when clinical conditions suggestive of post-infectious BO and specific HRCT scan abnormalities (mosaic perfusion, bronchiectasis) are present, once other possible causes of chronic obstructive pulmonary diseases have been excluded [1,7,22-24]. All 37 post-infectious BO patients in our study had histories of obstruction of the airways that persisted over 6 weeks after the initial event and had suggestive CT scans. Other diseases were excluded. Fourteen patients had open lung biopsies with pathologic findings consistent with obliterative bronchiolitis (2 with signs of aspiration).

Mauad et al. [26] studied the histopathological features of 34 pediatric patients with a diagnosis of $\mathrm{BO}$ and showed that childhood bronchiolitis obliterans is histologically characterized mainly by a constrictive pattern (97\%), which is in agreement with the findings in our sample (13 constrictive patterns from 18 biopsies).

Pulmonary function tests in children with post-infectious BO typically show severe airway obstruction with small responses to bronchodilation, increased resistance, normal or increased total lung capacity (TLC) and elevated residual volume (RV) due to hyperinflation and air trapping $[1,10,23,24]$. In our study, all of the tested subjects had patterns of pulmonary function characterized by mild to severe obstruction and significantly diminished $\mathrm{FEV}_{1}$.

There is no consensus on to the optimal treatment for BO $[6,8]$. General supportive measures include avoidance of tobacco smoke and other inhaled irritants, the use of influenza vaccination, chest physiotherapy, adequate nutritional intake and the administration of supplemental oxygen for hypoxemic patients $[1,10]$.

Although systemic corticosteroids are frequently used, their effectiveness in improving the outcomes for patients with $\mathrm{BO}$, as well as the best route of administration, is controversial $[1,23]$. Some clinicians opt for the systemic use of corticosteroids rather than delivery by inhalation because the obliterative lesions in the small airways can interfere with the deposition of aerosol $[8,23,25]$. Others prefer inhaled corticosteroids to minimize the adverse systemic effects and to reduce airway hyperreactivity $[6,19]$. 
The advantages of intravenous pulse therapy are the enhancement of the therapeutic effect and reduction of the side effects caused by the intermittent administration of high doses of drugs [27-29]. Corticosteroid pulse therapy is arbitrarily defined as more than $250 \mathrm{mg}$ prednisone or its equivalent per day [29]. The first reported use of a high-dose intravenous corticosteroid was in 1969, when it was used to successfully prevent renal allograft rejection [29]. Since then, high-dose intravenous corticosteroids have come to be used in a variety of inflammatory conditions [27-30].

In the field of pulmonology, pulse therapy has been effective in the treatment of other inflammatory lung diseases, such as chronic interstitial lung diseases [31,32]. There are limited reports of its use in patients with postinfectious BO [8]. Recently, it has been administered to pediatric patients with post-transplant BO. Ratjen et al. [33] studied nine children who were treated with pulse methylprednisolone therapy after being diagnosed with BO subsequent to bone marrow transplantation. The oxygen saturation in all of these individuals increased significantly and was normalized at the end of therapy. In 5 of the 9 patients, the treatment led to stabilization of the lung function without further deterioration during the follow-up period, but normalization of the pulmonary function was not achieved.

In our study, the children with BO who were treated with the high-dose methylprednisolone pulse therapy displayed clinical improvements as demonstrated by decreased wheezing exacerbations and improved oxygen saturation. The numbers of hospitalizations were reduced as a consequence. These data suggest that this therapy may be a valuable treatment option for children with $\mathrm{BO}$.

Twenty-four months after the beginning of the pulse therapy, $83 \%$ of patients who received prolonged oral corticosteroid therapy were able to stop this prolonged therapy. Twenty-five percent of hypoxemic patients completely discontinued supplemental oxygen therapy, and in another $25 \%$ of patients, oxygen therapy was modified from full-time administration to overnight-only. Approximately $52 \%$ of the patients with evidence of pulmonary hypertension by echocardiogram exhibited normalization following the pulse therapy. In our opinion, the most important reason for recommending pulse therapy in patients with $\mathrm{BO}$ should be a dependence on oral corticosteroid therapy and a need for domiciliary oxygen therapy.

Comparing patients with and without prolonged oral corticosteroid therapy, we found that the difference in pulse therapy duration was not significantly different. The group with oral corticosteroids had a decrease in the number of hospitalizations but not of exacerbations, indicating that the exacerbation severity was reduced but not the frequency. The group without oral corticosteroids had a decrease in the number of exacerbations but not hospitalizations. The median hospitalization before the pulse therapy was already zero, indicating that exacerbations were less severe in this group.

With regards to safety, the toxicity of corticosteroids is manifested mainly by disturbances in electrolytes, suppressed growth, loss of bone mass, cushingoid appearance, gastrointestinal bleeding, neural complications, metabolic toxicity, renal complications, cardiac toxicity, ophthalmic problems and damage to the immunological system, which result in an increased susceptibility to infections [27-30,34,35]. In patients with normal kidney function, the renal effects of pulse therapy seem to be minimal. However, in nephritic patients they can lead to renal deterioration [36].

In our sample, all the acute effects during the methylprednisolone intravenous infusion were transient, and none were serious. Hyperglycemia occurred in 9\% of cycles, hypernatremia in $1.7 \%$ and acute hypertension in $2.3 \%$.

In our study, 8 patients presented with osteoporosis (1 had hypophosphatemic rachitis with phosphoruslosing tubulopathy), 1 developed a cataract one year after pulse therapy, 7 had abnormal urinary tract ultrasounds ( 2 cases of nephrolithiasis, 4 cases of nephrocalcinosis and 1 case of small pyelocaliceal dilatation), and 16 presented with systemic hypertension ( 9 of whom exhibited this condition prior to pulse therapy). None of the patients were diagnosed with diabetes mellitus.

Nevertheless, the adverse effects of corticosteroids are dose and time-dependent, so it is impossible to discriminate between prolonged corticosteroid therapy side effects due to pulse therapy and/or those due to any oral corticosteroid that the patients received for wheezing exacerbations. It is important to note that 23 patients were already on continuous oral corticosteroid therapy at the beginning of this treatment.

There is a general concern regarding the effect of corticosteroids in pediatric patients. However, in our study, the pulse therapy did not appear to have interfered with growth. Indeed, we observed improvements in height and weight, possibly because of the clinical stabilization. After pulse therapy, the mean Z-score of length by age improved from -1.08 to -0.63 and the mean Z-score of weight by age improved from -0.91 to -0.59 .

As far as we know, this is the largest series of pediatric BO patients treated with methylprednisolone pulse therapy that has been reported to date. However, we are aware that the present study has a number of limitations. First, this study did not include a control group and therefore cannot definitively prove that treatment altered the natural course of the disease. Additionally, it is well known that the small airways are disproportionately 
narrow in the early years of life. However, lung growth increases peripheral airway conduction, which reduces airway resistance. The airways consequently become less vulnerable to obstruction [23]. Therefore, the clinical improvement observed in our sample of patients may be due to the physical increase in the sizes of the peripheral airways, which is the consequence of normal lung growth and does not necessarily represent regression of the pathology [19,23]. Finally, our cohort was relatively small because of the low frequency of this disease in pediatric patients. A multicenter approach would be needed to sample a larger patient cohort.

In contrast to the high mortality rate for posttransplantation BO (25-56\%) [4,37,38], post-infectious BO tends to show clinical improvement after 2-3 years of supportive therapy, although clinical, radiological and spirometric changes might persist $[8,19,23-25]$. As shown in a study by Zhang et al. [23] of 31 patients with postinfectious BO, clinical remission was found in $22.6 \%$ of the patients, $67.7 \%$ had persistent respiratory signs and symptoms, and $9.7 \%$ of the patients died. In another study of 20 post-infectious $\mathrm{BO}$ patients [25], no improvement was evident in $40 \%$ of patients, $55 \%$ had partial remission of symptoms, 5\% died of the disease and none recovered completely after 3 years of follow-up. In a Brazilian study of 48 patients with post-infectious $\mathrm{BO}$ [39], there was partial clinical improvement in $65 \%$ of patients, but $35 \%$ had no improvement at all after 3 years of follow-up.

However, the most worrisome results were found in the study by Cazzato et al. [40]. In this study, eleven patients with post-infectious $\mathrm{BO}$ were followed up for a mean period of 10.2 years, and $\mathrm{FEV}_{1}$ and $\mathrm{FEF}_{25-75 \%}$ fell at rates of $-1.01 \%$ and $-1.04 \%$ per year, respectively. These results suggest that childhood post-infectious BO can be a progressive lung disorder with increasing lung function impairment. Consequently, patients may need an anti-inflammatory treatment to prevent the progression of the lesions.

\section{Conclusions}

In conclusion, our results suggest that intravenous methylprednisolone pulse therapy appears to be useful and fairly safe in pediatric patients with bronchiolitis obliterans. This treatment can modify the progress of the disease and may be an alternative option for administering systemic corticosteroids and thereby avoiding the side effects associated with continuous oral corticotherapy. Further prospective controlled studies are necessary to confirm these findings.

\section{Abbreviations}

BO: Bronchiolitis obliterans; HRCT: High Resolution Computed Tomography of the lungs; FVC: Forced vital capacity; FEV 1 : Forced expiratory volume in one second; $\mathrm{FEF}_{25-75 \%}$ : Forced expiratory flow between $25 \%$ and $75 \%$ of FVC.
Competing interests

The authors declare that they have no competing interests.

\section{Authors' contributions}

SOT collected data, participated in the design of the study and helped to draft the manuscript; FVA and LVRFdSF participated in the design of the study; CL performed the statistical analysis; JCR conceived of the study, participated in its design and coordination and helped to draft the manuscript. All authors revised critically and approved the final manuscript.

\section{Acknowledgements}

We appreciate the help of Dr. Ulysses Doria Filho for his assistance in reviewing the statistical analysis.

\section{Author details}

${ }^{1}$ Pediatric Pulmonology Division, Instituto da Criança, Hospital das Clínicas, University of São Paulo, Avenida Dr Enéas de Carvalho Aguiar, 647, CEP 05403-000 São Paulo, SP, Brazil. ²Department of Maternal and Child Health, College of Public Health, University of São Paulo, Avenida Dr Arnaldo, 715, CEP 01246-904 São Paulo, SP, Brazil. ${ }^{3}$ Rua Bianchi Bertoldi, 166 apt 101, CEP: 05422-070 São Paulo, SP, Brazil.

Received: 27 March 2014 Accepted: 4 August 2014

Published: 15 August 2014

\section{References}

1. Moonnumakal SP, Fan LL: Bronchiolitis obliterans in children. Curr Opin Pediatr 2008, 20:272-278

2. Colom AJ, Teper AM, Vollmer WM, Diette GB: Risk factors for the development of bronchiolitis obliterans in children with bronchiolitis. Thorax 2006, 61:503-506.

3. Hardy KA, Schidlow DV, Zaeri N: Obliterative bronchiolitis in children. Chest 1988, 93:460-466.

4. Kurland G, Michelson P: Bronchiolitis obliterans in children. Pediatr Pulmonol 2005, 39:193-208.

5. Zhang L, Silva FA: Bronchiolitis obliterans in children. J Pediatr (Rio J) 2000, 76:185-192

6. Lobo AL, Guardiano M, Nunes T, Azevedo I, Vaz LG: Pos-infectious bronchiolitis obliterans in children. Rev Port Pneumol 2007, 13:495-509.

7. Jones MH, Pitrez PM, Stein RT: Post-infectious bronchiolitis obliterans. Pediatr Pulmonol Suppl 2004, 26:64-65.

8. Kim CK, Kim SW, Kim JS, Koh YY, Cohen AH, Deterding RR, White CW: Bronchiolitis obliterans in the 1990s in Korea and the United States. Chest 2001, 120:1101-1106.

9. Moran TJ, Hellstrom HR: Bronchiolitis obliterans: an experimental study of the pathogenesis and the use of cortisone in modification of the lesions. AMA Arch Pathol 1958, 66:691-707.

10. Teper A, Fischer $G B$, Jones $M H$ : Respiratory sequelae of viral diseases: from diagnosis to treatment. J Pediatr (Rio J) 2002, 78(Suppl 2):S187-S194.

11. Kanai H, Sawanobori E, Kobayashi A, Matsushita K, Sugita K, Higashida K: Early treatment with methylprednisolone pulse therapy combined with tonsillectomy for heavy proteinuric henoch-schönlein purpura nephritis in children. Nephron Extra 2011, 1:101-111.

12. Mori K, Honda M, Ikeda M: Efficacy of methylprednisolone pulse therapy in steroid-resistant nephrotic syndrome. Pediatr Nephrol 2004, 19:1232-1236

13. Ogata S, Ogihara Y, Honda T, Kon S, Akiyama K, Ishii M: Corticosteroid pulse combination therapy for refractory Kawasaki disease: a randomized trial. Pediatrics 2012, 129:e17-e23.

14. Miura M, Tamame T, Naganuma T, Chinen S, Matsuoka M, Ohki H: Steroid pulse therapy for Kawasaki disease unresponsive to additional immunoglobulin therapy. Paediatr Child Health 2011, 16:479-484.

15. Adebajo AO, Hall MA: The use of intravenous pulsed methylprednisolone in the treatment of systemic-onset juvenile chronic arthritis. Br J Rheumatol 1998, 37:1240-1242.

16. You SY, Jwa HJ, Yang EA, Kil HR, Lee JH: Effects of methylprednisolone pulse therapy on refractory mycoplasma pneumoniae pneumonia in children. Allergy Asthma Immunol Res 2014, 6:22-26. 
17. Miller MR, Hankinson J, Brusasco V, Burgos F, Casaburi R, Coates A, Crapo R, Enright P, Van der Grinten CP, Gustafsson P, Jensen R, Johnson DC, Maclntyre N, McKay R, Navajas D, Pedersen OF, Pellegrino R, Viegi G, Wanger J, ATS/ERS Task Force: Standardisation of spirometry. Eur Respir J 2005, 26:319-338.

18. Guidelines of the Brazilian Society of Cardiology: Diagnosis, evaluation and treatment of pulmonary hypertension; 2005 [http://publicacoes.cardiol.br/ consenso/2005/039.asp]

19. Chiu CY, Wong KS, Huang YC, Lin TY: Bronchiolitis obliterans in children: clinical presentation, therapy and long-term follow-up. J Paediatr Child Health 2008, 44:129-133.

20. Murtagh P, Kajon A: Chronic pulmonary sequelae of adenovirus infection. Pediatr Pulmonol Suppl 1997, 16:150-151.

21. Castro-Rodriguez JA, Daszenies C, Garcia M, Meyer R, Gonzales R: Adenovirus pneumonia in infants and factors for developing bronchiolitis obliterans: a 5-year follow-up. Pediatr Pulmonol 2006, 41:947-953

22. Chan PW, Muridan R, Debruyne JA: Bronchiolitis obliterans in children: clinical profile and diagnosis. Respirology 2000, 5:369-375.

23. Zhang L, Irion K, Kozakewich H, Reid L, Camargo JJ, Da Silva Porto N, Abreu e Silva FA: Clinical course of postinfectious bronchiolitis obliterans. Pediatr Pulmonol 2000, 29:341-350.

24. Chang AB, Masel JP, Masters B: Post-infectious bronchiolitis obliterans: clinical, radiological and pulmonary function sequelae. Pediatr Radiol 1998, 28:23-29.

25. Yalcin E, Dogru D, Haliloglu M, Ozcelik U, Kiper N, Gocmen A: Postinfectious bronchiolitis obliterans in children: clinical and radiological profile and prognostic factors. Respiration 2003, 70:371-375.

26. Mauad T, Dolhnikoff M: Histology of childhood bronchiolitis obliterans. Pediatr Pulmonol 2002, 33:466-474.

27. Hari P, Srivastava RN: Pulse corticosteroid therapy with methylprednisolone or dexamethasone. Indian J Pediatr 1998, 65:557-560

28. Roujeau JC: Pulse glucocorticoid therapy: the 'big shot' revisited. Arch Dermatol 1996, 132:1499-1502.

29. Sinha A, Bagga A: Pulse steroid therapy. Indian J Pediatr 2008, 75:1057-1066

30. Sabir S, Werth VP: Pulse glucocorticoids. Dermatol Clin 2000, 18:437-446

31. Desmarquest P, Tamalet A, Fauroux B, Boule M, Boccon-Gibod L, Tournier G, Clement A: Chronic interstitial lung disease in children: response to high-dose intravenous methylprednisolone pulses. Pediatr Pulmonol 1998, 26:332-338.

32. Clement A: Task force on chronic interstitial lung disease in immunocompetent children. Eur Respir J 2004, 24:686-697.

33. Ratjen F, Rjabko O, Kremens B: High-dose corticosteroid therapy for bronchiolitis obliterans after bone marrow transplantation in children. Bone Marrow Transplant 2005, 36:135-138.

34. Wollheim FA: Acute and long-term complications of corticosteroid pulse therapy. Scand I Rheumatol 1984, 54(Suppl):27-32.

35. Damiani D, Kuperman H, Dichtchekenian V, Manna TD, Setian D: Corticoterapia e suas repercussões: a relação custo-benefício. Pediatria (Säo Paulo) 2001, 23:71-82.

36. Sakemi T, Yamaguchi M, Fujimi S, Nagano Y, Uchida M: Effects of the methylprednisolone pulse therapy on renal function. Am J Nephrol 1991 11:48-53.

37. Griese M, Rampf U, Hofmann D, Fuhrer M, Reinhardt D, Der-Gotze C: Pulmonary complications after bone marrow transplantation in children: twenty-four years of experience in a single pediatric center. Pediatr Pulmonol 2000, 30:393-401.

38. Chan A, Allen R: Bronchiolitis obliterans: an update. Curr Opin Pulm Med 2004, 10:133-141.
39. dos Santos RV, Rosario NA, Ried CA: Bronquiolite obliterante pós infecciosa: aspectos clínicos e complementares de 48 crianças. J Bras Pneumol 2004, 30:20-25.

40. Cazzato S, Poletti V, Bernardi F, Loroni L, Bertelli L, Colonna S, Zappulla F, Timoncini G, Cicognani A: Airway inflammation and lung function decline in childhood post-infectious bronchiolitis obliterans. Pediatr Pulmonol 2008, 43:381-390.

doi:10.1186/s13023-014-0128-2

Cite this article as: Tomikawa et al:: Follow-up on pediatric patients with bronchiolitis obliterans treated with corticosteroid pulse therapy.

Orphanet Journal of Rare Diseases 2014 9:128

\section{Submit your next manuscript to BioMed Central and take full advantage of:}

- Convenient online submission

- Thorough peer review

- No space constraints or color figure charges

- Immediate publication on acceptance

- Inclusion in PubMed, CAS, Scopus and Google Scholar

- Research which is freely available for redistribution

Submit your manuscript at www.biomedcentral.com/submit
Biomed Central 